\title{
Analysis of Fatigue Life of PMMA at Different Frequencies Based on a New Damage Mechanics Model
}

\author{
Aifeng Huang, ${ }^{1}$ Weixing Yao, ${ }^{2}$ and Fang Chen $^{2}$ \\ ${ }^{1}$ Research Institute of Unmanned Aircraft, Nanjing University of Aeronautics and Astronautics, Nanjing 210016, China \\ ${ }^{2}$ State Key Laboratory of Mechanics and Control of Mechanical Structures, Nanjing University of Aeronautics and Astronautics, \\ Nanjing 210016, China
}

Correspondence should be addressed to Weixing Yao; wxyao@nuaa.edu.cn

Received 12 March 2014; Revised 28 April 2014; Accepted 4 May 2014; Published 11 June 2014

Academic Editor: Jose Merodio

Copyright (C) 2014 Aifeng Huang et al. This is an open access article distributed under the Creative Commons Attribution License, which permits unrestricted use, distribution, and reproduction in any medium, provided the original work is properly cited.

\begin{abstract}
Low-cycle fatigue tests at different frequencies and creep tests under different stress levels of Plexiglas Resist 45 were conducted. Correspondingly, the creep fracture time, $S$ - $N$ curves, cyclic creep, and hysteresis loop were obtained. These results showed that the fatigue life increases with frequency at low frequency domain. After analysis, it was found that fatigue life is dependent on the load rate and is affected by the creep damage. In addition, a new continuum damage mechanics (CDM) model was established to analyze creep-fatigue life, where the damage increment nonlinear summation rule was proposed and the frequency modification was made on the fatigue damage evolution equation. Differential evolution (DE) algorithm was employed to determine the parameters within the model. The proposed model described fatigue life under different frequencies, and the calculated results agreed well with the experimental results.
\end{abstract}

\section{Introduction}

Because of its high impact resistance, sufficient strength, and excellent transmittance, PMMA material has wide application. Since many structures must withstand cyclic loading during their service lives, the research on fatigue of PMMA is attracting an increased interest [1-3].

PMMA is a typical kind of viscoelastic material. The fatigue properties of viscoelastic materials are sensitive to temperature and cyclic frequency, which is one of the biggest differences from conventional metal material. This paper is concerned with the frequency sensitivity. Lots of researchers have investigated the fatigue property of PMMA and other polymers at different frequencies. Cheng et al. [2] conducted PMMA fatigue crack propagation (FCP) experiments at frequencies of $1,10,50$, and $100 \mathrm{~Hz}$. Test temperature ranged from -30 to $100^{\circ} \mathrm{C}$. Their results showed that FCP rates decrease as cyclic frequency increases until temperature approaches the glass transition region. Jia et al. [4] and Feng et al. [5] performed fatigue tests on PMMA used in aircraft and ordinary industrial grade; they all observed that FCP rates decrease as cyclic frequency increases. Luo et al. [6] carried out several fatigue tests of YB-3 PMMA under the frequency of $0.1,1$, and $10 \mathrm{~Hz}$; the results showed that fatigue life increased with increasing frequency, although the increase was not so notable in high frequency range. Wang et al. [7] also conducted PMMA fatigue tests at the frequency of 1,2 , and $5 \mathrm{~Hz}$, and the difference was that fatigue life was observed to decrease with frequency except at high stress level.

The fatigue life of PMMA varies with frequency indisputably, and that its fatigue crack propagation rate decreases with frequency is a consistent conclusion of most experiments. However, there is not such consistent relationship between fatigue life and loading frequency. Many research analyzed the influence mechanism of frequency on fatigue behavior. It can be summed up to three attributions: hysteretic heating, creep, and rate sensitivity. Under cyclic loading, the hysteresis loop is formed by out of phase stresses and strains due to high internal damping of polymers. Hysteresis loop indicates energy dissipation or heat generation named hysteretic heating. If frequency is high, hysteretic heat cannot be dissipated rapidly enough because of low thermal conductivity of polymers, and thus temperature will rise up. Hysteretic 
TABLE 1: Typical property values (at $23^{\circ} \mathrm{C}$ and $50 \%$ relative humidity) of Plexiglas Resist 45.

\begin{tabular}{lccccc}
\hline Density & Transmittance & Tensile strength & $\begin{array}{c}\text { Modulus of } \\
\text { elasticity } \\
\text { (short-term value) }\end{array}$ & Poisson's rate & $\begin{array}{c}\text { Nominal } \\
\text { elongation at break }\end{array}$ \\
\hline $1.19 \mathrm{~g} / \mathrm{cm}^{3}$ & $91 \%$ & $60 \mathrm{MPa}$ & $2700 \mathrm{MPa}$ & 0.41 & $10 \%$ \\
\hline
\end{tabular}

heating will soften the material, and both stiffness and strength will decrease. This is the reason for fatigue life decreasing with frequency in some experiments. Creep phenomenon is significant and common in polymers. During fatigue process with nonzero mean stress, creep deformation and creep damage can be observed apparently even without load holding. More creep damage accumulates in one cycle under lower frequency for longer time per cycle. This will result in decrement of fatigue life as frequency decreases. Mechanical properties of viscoelastic material are dependent upon strain rate. For polymers, strain rate effect is an important property as creep. In unidirectional tension or compression and impact research field, strain rate effect has been paid quite more attention. Strain rate hardening and strain rate strengthening phenomenon of PMMA were observed in lots of experiments $[8,9]$. It is well reasoned to deduce that frequency is higher, strain rate is necessarily higher, and then stiffness and tensile strength will be higher, and fatigue strength will be higher too. That is to say, at the same load level, frequency is higher, and fatigue life will be larger. Unfortunately, rate effect in PMMA fatigue was only mentioned occasionally; the attention paid to rate effect was far from enough. In general, fatigue life variation with frequency is related to frequency range, stress level, and other parameters and is determined by the dominate mechanism. For example, hysteretic heat is notable at high frequency, creep damage contributes more during lower frequency, and rate effect may play a part in a wide frequency range.

Hysteretic heating in polymers was noticed and investigated from an early period; many works have been done [10, 11]. For creep damage, equations similar to Paris law including fatigue and creep have been proposed to describe the FCP rate [12, 13]. Luo et al. [6] established a creep-fatigue life model based on continuum damage mechanics. However, rate effect consideration appears lacking in the fatigue life prediction model.

The authors of this paper performed fatigue test at 0.03 , 0.1 , and $0.5 \mathrm{~Hz}$ with Plexiglas Resist 45 and recorded its mechanical response at different frequencies to accumulate test data towards a better understanding of the frequency influence on PMMA fatigue behavior and relevant theoretical research. It is found that the temperature rise is insignificant at frequencies no more than $0.5 \mathrm{~Hz}$ [1]. Load frequency in this paper was low enough to take no account of hysteretic heating. Thus the problem could be simplified to investigate pure frequency influence. To assess creep damage during the fatigue process, creep tests were carried out. Moreover, a modified CDM creep-fatigue model was proposed to describe fatigue life at low frequency. The proposed model can also be used in the condition with load holding.

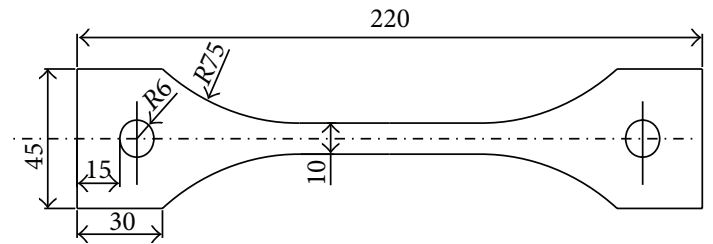

(a)

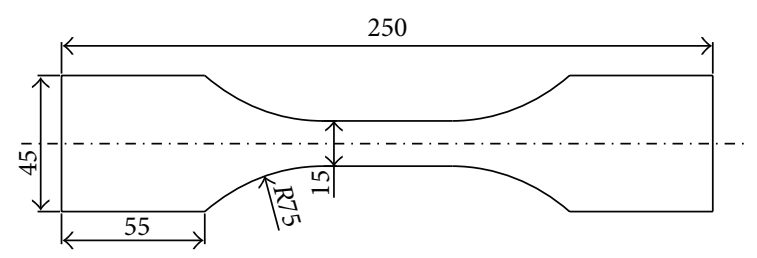

(b)

FIGURE 1: Geometry and dimensions (in $\mathrm{mm}$ ) of specimen: (a) creep specimen and (b) fatigue specimen.

\section{Experimental}

2.1. Material and Specimen. Plexiglas Resist 45 sheet, which is developed and produced by Röhm Company, is a kind of solid plastic sheet extruded from impact-modified PMMA. Some material properties of Plexiglas Resist 45 are presented in Table 1 .

The creep and fatigue specimens (see Figure 1) are $3 \mathrm{~mm}$ thick and are designed according to the standard of high polymers mechanics test [14].

2.2. Creep Test. In this paper, creep tests were carried out under constant load. To reduce test cost, creep tests were carried out on the self-designed equipment which apply constant load by hanging the weight. A camera was utilized to monitor and record the fracture time. A gauge length with an initial value of about $55 \mathrm{~mm}$ was drawn on the specimen beforehand. In some interval, the gauge length was measured by microcaliper. Although the error of this measuring method was big, the approximate shape of creep curve could be obtained because of big creep deformation of specimen. Five levels of nominal stress were used in creep tests: $30 \mathrm{MPa}$, $33 \mathrm{MPa}, 36 \mathrm{MPa}, 37 \mathrm{MPa}$, and $40 \mathrm{MPa}$. Three specimens were tested at each stress level.

2.3. Fatigue Test. Fatigue tests were run by applying sinusoidal tensile loads with constant amplitude, in load control mode, on a MTS809 servohydraulic testing machine. The environment temperature was controlled at about $27^{\circ} \mathrm{C}$ 


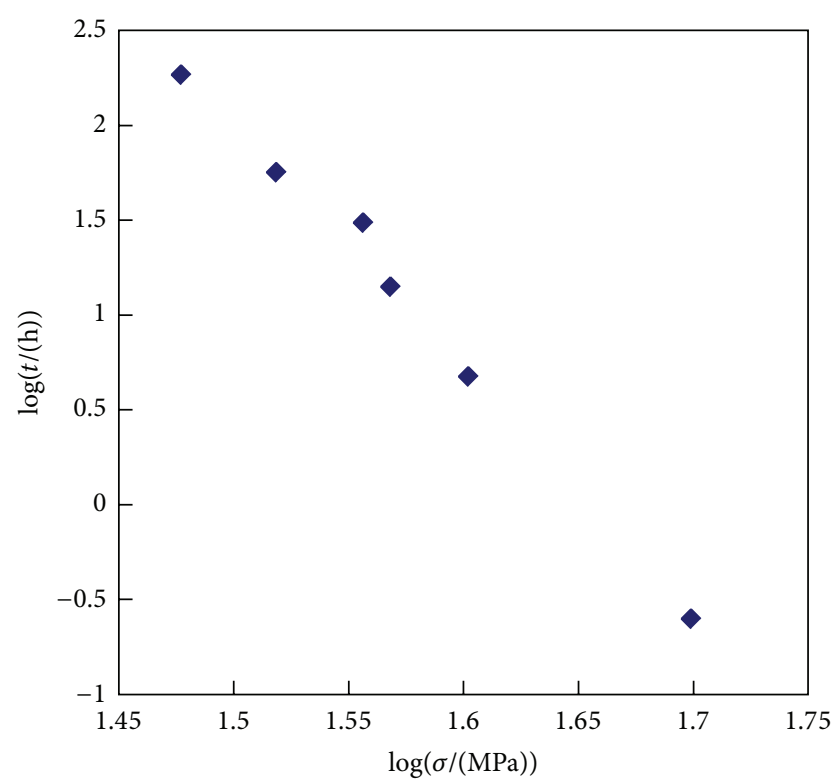

FIGURE 2: Creep fracture time at different stresses.

throughout all tests. 30, 33, 39, 42, and $44 \mathrm{MPa}$ were, respectively, chosen as maximum stress. Three series of tests were conducted with cyclic load frequency values of $0.03,0.1$, and $0.5 \mathrm{~Hz}$. To prevent specimens from buckling under compressive loading, tension to tension load cycles were applied with a load ratio 0.01 for all tests. During the load controlled fatigue tests, in order to observe cyclic creep and hysteresis loop, axial strains were measured by MTS632.13F-20 extensometer with $10 \mathrm{~mm}$ base length. Full stress strain cycles were recorded at fixed time intervals.

\section{Results and Discussions}

3.1. Creep Result. The creep curve of Plexiglas Resist 45 was composed of three typical stages, with rapid strain rate in initial stage and before fracturing. The fracture time was presented in Figure 2, where the log (fracture time) was plotted versus $\log$ (stress). The fracture time covered 4 hours to 200 hours and decreased steeply with increasing stress. As shown in Figure 2, the data points were nearly in a line.

\subsection{Fatigue Test Result}

3.2.1. Fatigue Life. The results of fatigue tests were reported in Figure 3, where $\sigma_{\max }$ was plotted versus $\log N$. These three curves were far from coincidence, with fatigue life increasing with increasing cyclic frequency.

3.2.2. Cyclic Creep. Hysteresis loop is always observed to move gradually along the strain axis during nonzero mean stress fatigue process of polymers, and this indicates that cyclic creep strain accumulates gradually. For different materials, load holding time or frequency has a different effect on cyclic creep rate. For some high polymers, frequency exhibits an acceleration effect on cyclic creep rate [15]. This paper

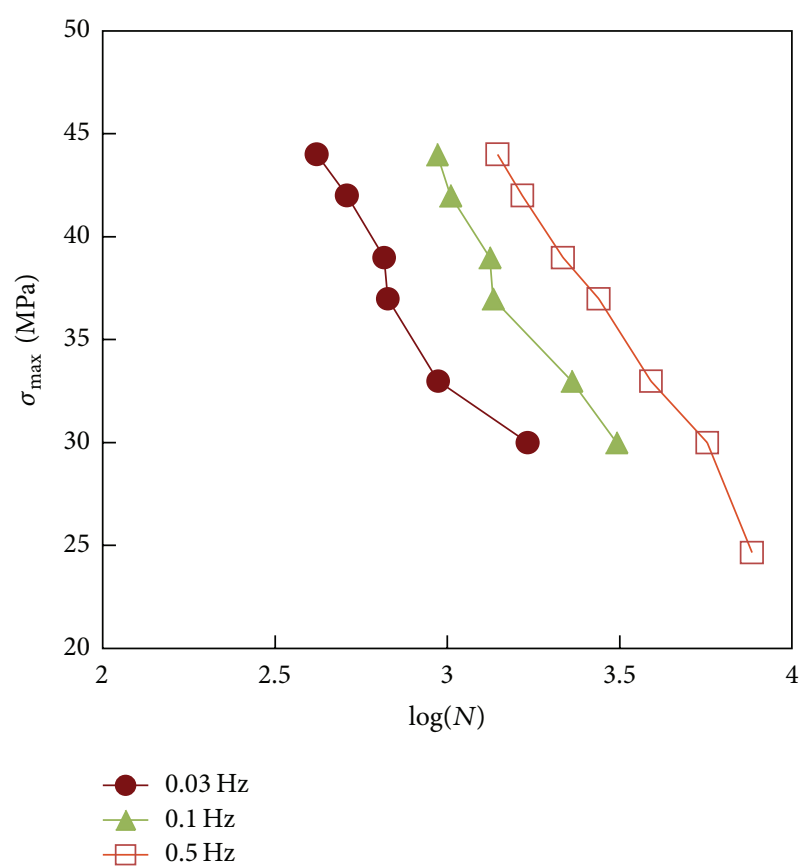

Figure 3: Fatigue life and $S$ - $N$ curve at different frequencies.

attempted to observe the cyclic creep of Plexiglas Resist 45 at different frequencies. Because the stress ratio of fatigue test in this paper was close to zero $(R=0.01)$, the elastic component included in minimum ( $\mathrm{min}$ ) displacement was very small; the min displacement could be treated approximately as cyclic creep displacement. The min displacements at different frequencies were presented in Figure $4\left(\sigma_{\max }=33 \mathrm{MPa}\right)$ and Figure $5\left(\sigma_{\max }=44 \mathrm{MPa}\right)$. The results at other stress levels were similar. It could be seen from Figures 4(a) and 5(a) that frequency slightly accelerated the cyclic creep rate of the first stage. For the second stage of the major part of cyclic creep curve, frequency had no notable acceleration effect. The comparisons between Figures 4(a) and 4(b) and Figures 5(a) and 5(b) demonstrated that cyclic creep was more related to time than to cycles.

3.2.3. Hysteresis Loop. Because mechanical properties of viscoelastic material such as elastic modulus and viscous damp are related to strain rate, hysteresis loop at different frequencies exhibits some differences. This paper investigated the frequency influence on hysteresis loop. The hysteresis loop of $\sigma_{\max }=39 \mathrm{MPa}$ at half-life was presented in Figure 6. The results at other stress levels were similar. The area within hysteresis loop was calculated by integration. The integration results showed that the area did not vary with frequency significantly. The test results by Wang et al. [7] on Plexiglas also demonstrated that frequency did not notably affect the area of the hysteresis loop. However, as seen in Figure 6, the "slope" of hysteresis loop decreased with frequency. Modulus $E$ was defined as $E=\Delta \sigma / \Delta \varepsilon$, and the calculated $E$ was dotted in Figure 7 where a fitted power functional curve of $E$ was plotted. As shown in Figure 7, modulus increased with frequency 


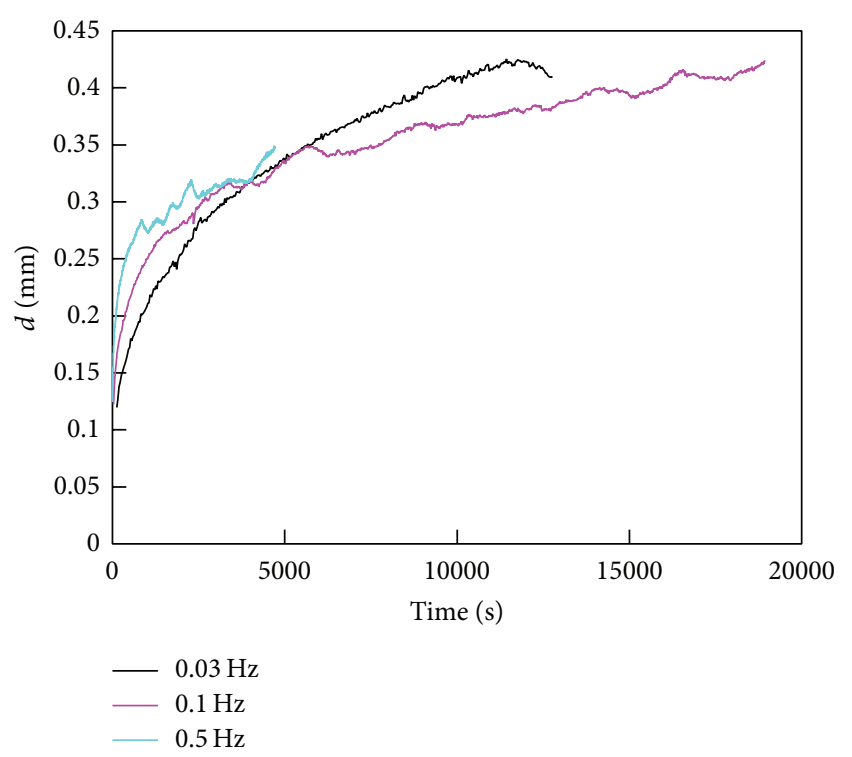

(a)
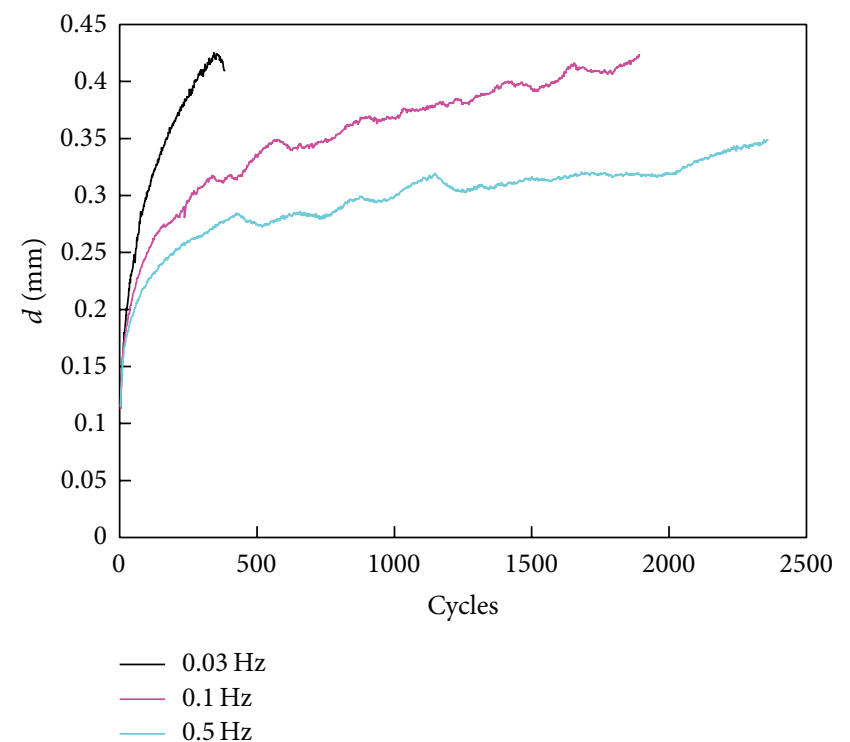

(b)

Figure 4: (a) Min displacement versus time and (b) min displacement versus cycles at different frequencies $\left(\sigma_{\max }=33 \mathrm{MPa}\right)$.

especially at low frequency, and $E$ could be expressed as the power function of frequency.

\section{CDM Model for Fatigue Life Prediction}

Because the continuum damage mechanics approach can cope with the coexisting of multiform damage, it is a suitable and promising method for creep-fatigue life prediction. Luo et al. [6] have established the creep-fatigue damage model of YB-3 Plexiglas based on CDM; additionally the pure fatigue damage has been separated from the fatigue test including creep damage. This paper stepped forward on their work.
The main work of CDM approach to prediction fatigue life includes establishment of damage evolution equation and determination of the initial and critical damage value. For the sake of convenience, damage initial value is mostly taken as zero $D_{0}=0$ and critical value as unity $D_{\text {cr }}=1$.

4.1. Frequency Modified Fatigue Damage Evolution Equation. The following formula is a constantly used fatigue damage evolution under simple load condition [16]:

$$
\frac{\mathrm{d} D}{\mathrm{~d} N}=\frac{\sigma_{a}^{2 s+1}}{B(1-D)^{2 s}},
$$

where $s$ and $B$ are material constants, $D$ is the damage variable, and $\sigma_{a}$ is stress amplitude.

The above analysis has concluded that, because mechanical properties of viscoelastic material are related to strain rate, fatigue strength would increase with frequency. But the quantity relationship of fatigue strength to frequency is difficult to determine. Experimental results in [9] showed that initial modulus along with tensile strength increases with strain rate, and they both have similar functional relationship with strain rate. It should be possible that functional relationship between fatigue strength and frequency is similar to modulus and frequency. According to results in [17] and this paper, the modulus increases with frequency significantly when frequency is low and then tends to be stable at higher frequency. Figure 7 showed that modulus could be expressed as power function of frequency in low frequency range. Hence, this paper proposed the frequency modified fatigue damage evolution equation expressed as

$$
\frac{\mathrm{d} D}{\mathrm{~d} N}=\frac{\sigma_{a}^{2 s+1}}{B f^{m}(1-D)^{2 s}},
$$

where $f$ is cyclic frequency and $m$ is constant. Because creep damage exists inevitably in fatigue process and load frequency of high polymers cannot be too high, it is difficult to obtain pure fatigue life directly from fatigue test. The parameters in (2) would be determined through later fitting calculation of creep-fatigue life.

4.2. Creep Damage Evolution Equation. As can be seen from cyclic creep result, the shape of cyclic creep curve was similar to static creep, and frequency did not accelerate the cyclic creep rate significantly. It can be assumed that creep under cyclic load may occur due to the same mechanism as static creep. Static creep damage evolution equation could be employed to describe the pure creep damage in fatigue process. Therefore, Kachanov equation was utilized to describe the creep damage evolution of Plexiglas Resist 45 and this equation has been proposed for a long time and has been verified in many aspects [18]:

$$
\frac{\mathrm{d} D}{\mathrm{~d} t}=A\left(\frac{\sigma}{1-D}\right)^{r}
$$

where $A$ and $r$ are material constants, which can be obtained through creep test. 


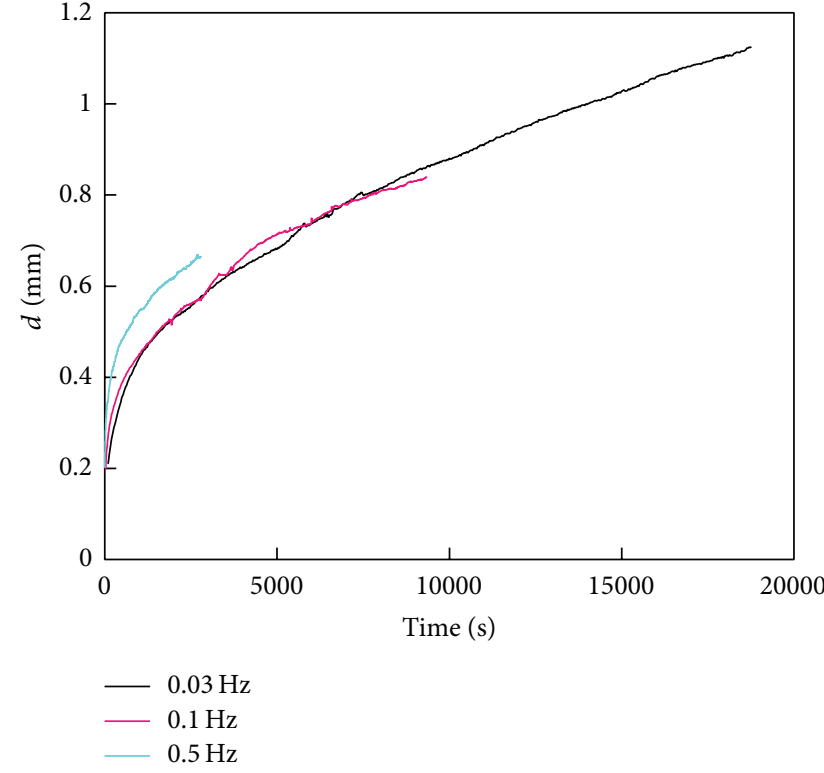

(a)

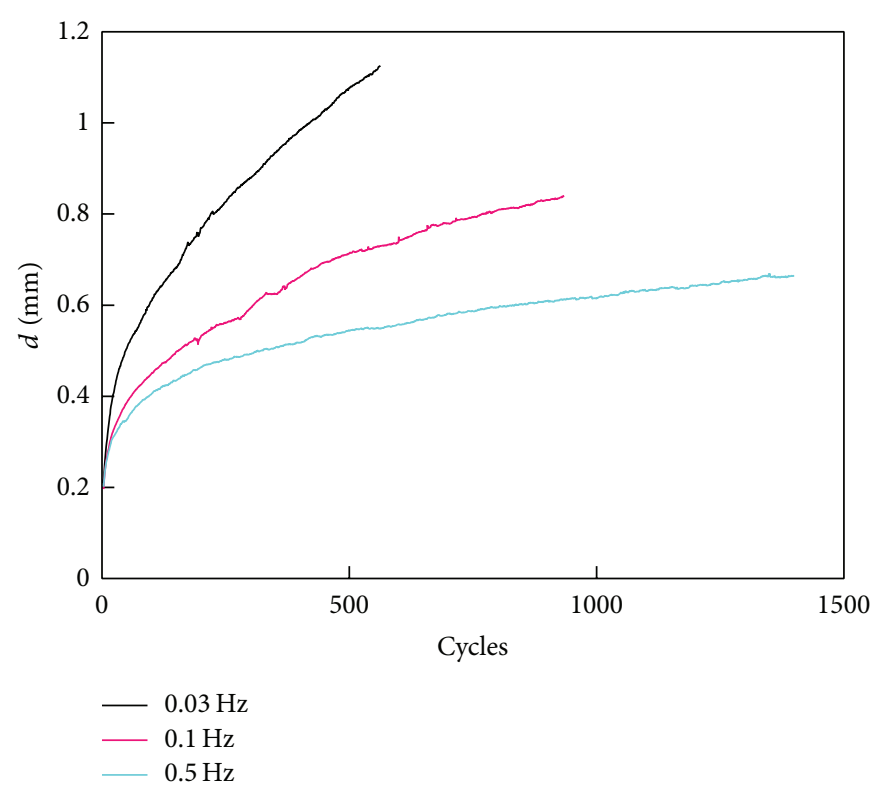

(b)

Figure 5: (a) Min displacement versus time and (b) min displacement versus cycles at different frequencies $\left(\sigma_{\max }=44 \mathrm{MPa}\right)$.

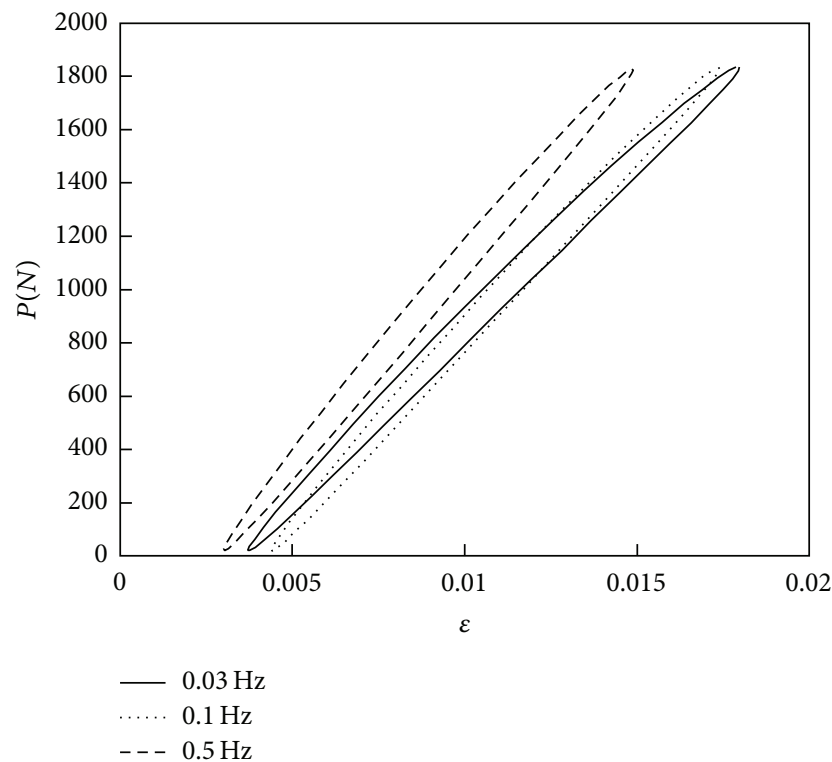

FIGURE 6: Hysteresis loop at different frequencies $\left(\sigma_{\max }=39 \mathrm{MPa}\right)$.

Integrating (3) with initial condition $\left.D\right|_{t=0}=0$, damage equation can be obtained as follows:

$$
D=1-\left(1-A(r+1) \sigma^{r} t\right)^{1 /(r+1)} .
$$

When material is failure, $D=1$, then fracture time $t_{c}$ can be written as

$$
t_{c}=\frac{1}{A(r+1)} \sigma^{-r}
$$

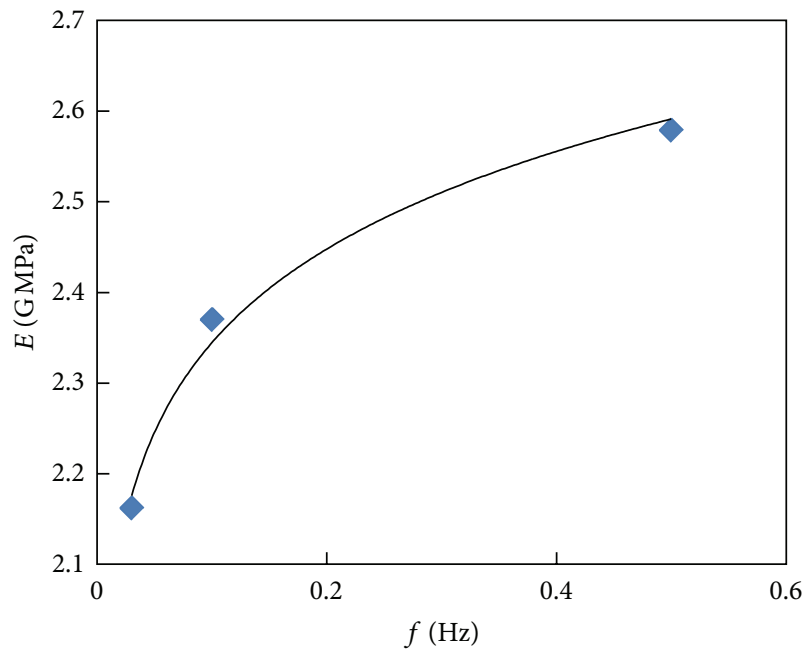

FIGURE 7: Modulus versus frequency $\left(\sigma_{\max }=39 \mathrm{MPa}\right)$.

Rewrite (5) in the following logarithm form:

$$
\log \left(t_{c}\right)=-\log (A(r+1))-r \log (\sigma) .
$$

Equation (6) showed that fracture time should be logarithm linear with stress level. $A=9.198 E-26, r=14.216$ could be determined from creep test results, and the units of $\sigma, t$, and $t_{c}$ are $\mathrm{MPa}$, hour, and hour, respectively.

4.3. Creep-Fatigue Damage Evolution Equation. For high polymers, creep damage and fatigue damage most often accumulate nonlinearly because of creep-fatigue interaction during creep-fatigue process $[15,19]$. To the creep-fatigue interaction question, Lemaitre and Chaboche presented a general kinetic 
equation for creep-fatigue damage problems based on the assumption that the total damage increment $\mathrm{d} D$ is the sum of fatigue damage increment $\mathrm{d} D_{f}$ and creep damage increment $\mathrm{d} D_{c}$ (i.e., $\left.\mathrm{d} D=\mathrm{d} D_{c}+\mathrm{d} D_{f}\right)[18]$. This model has been used with success during the past 30 years on various classes of materials and applications [20, 21]. Factually, the damage increment linear summation rule was not flawless; for example, Chaboche pointed out that this rule would overestimate creep-fatigue interaction $[22,23]$.

In most cases, creep-fatigue interaction will be strong when creep damage is nearly equal to fatigue damage and will be weak when either creep or fatigue is dominant. The interaction intensity in a short time or in one cycle should obey the same rule. Thus increment nonlinear summation rule was proposed here; in detail, fatigue damage is composed by fatigue damage itself and fatigue damage caused by creep, and in the same way creep damage is the sum of creep damage itself and creep damage caused by fatigue damage. The interaction damage is related to the relative quantity of creep damage and fatigue damage. When the sum of creep damage and fatigue damage attains critical value 1 , the material will fail:

$$
\begin{gathered}
\mathrm{d} D_{f}^{\prime}=\mathrm{d} D_{f}+C \cdot \mathrm{d} D_{c}, \\
\mathrm{~d} D_{c}^{\prime}=\mathrm{d} D_{c}+C \cdot \mathrm{d} D_{f}, \\
D_{f}=D_{f}+\mathrm{d} D_{f}^{\prime}, \\
D_{c}=D_{c}+\mathrm{d} D_{c}^{\prime}, \\
D_{\text {tot }}=D_{c}+D_{f},
\end{gathered}
$$

where $C=c^{\prime} \exp \left(2 \sqrt{\mathrm{d} D_{f} \cdot \mathrm{d} D_{c}} /\left(\mathrm{d} D_{f}+\mathrm{d} D_{c}\right)\right), c^{\prime}$ is a slightadjustment parameter and has a value of about 1 . Let $C \equiv 1$; damage increment linear summation rule will be obtained. Let $a=\mathrm{d} D_{c} / \mathrm{d} D_{f}$; then $C$ can be written as $C=$ $c^{\prime} \exp (2 \sqrt{a} /(1+a))$. Function $C$ is symmetric to $a=1$; that is, $C(a)=C(1 / a)$. The value of $\exp (2 \sqrt{a} /(1+a))$ varies from 1 to 2.718 and reaches maximum value at $a=1\left(\mathrm{~d} D_{f}=\mathrm{d} D_{c}\right)$. In most cases, the value is slightly greater than 1 , and this indicates that there is no big difference between the proposed damage summation rule and the widely used damage increment linear summation rule. But the expression of $C$ in this paper is more flexible and is consistent with the general rule that interaction is strongest when creep damage and fatigue damage are in the same level. Variable value $C$ along with slight-adjustment coefficient $c^{\prime}$ can make the calculated result agree better with experimental result.

Substituting (2) and (3) into (7) and (8), respectively, creep-fatigue damage evolution equation would be obtained. Generally, one cycle is chosen as integrate step size. $D_{c}$ is taken as constant value during one cycle; $\mathrm{d} D_{c}$ obtained by integrating (3) in one cycle is substituted to (8) then.

4.4. Parameter Determination. It is difficult to determine the parameters in creep-fatigue damage model. Differential evolution (DE) algorithm was employed to solve this problem. Parameters determined by DE were presented in Table 2. DE

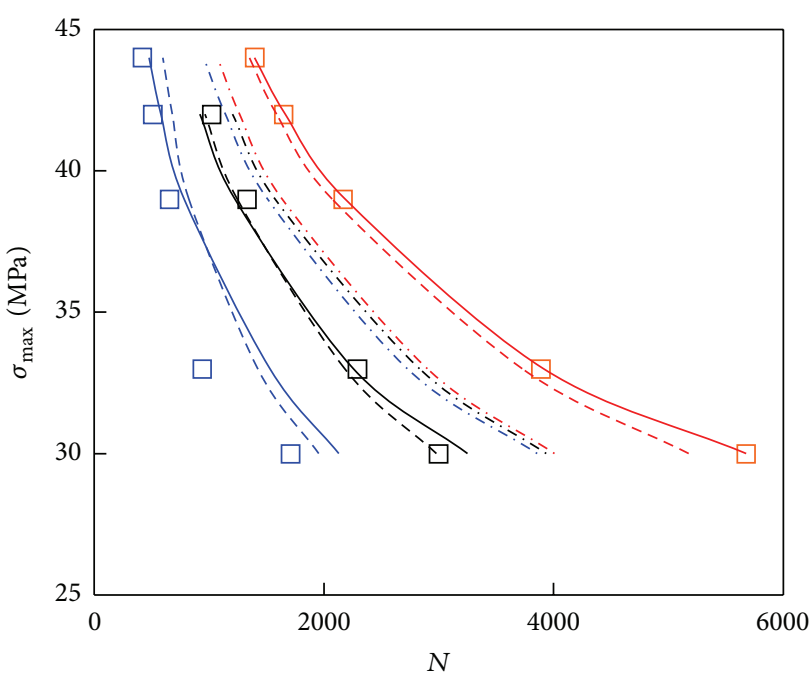

Figure 8: Fatigue life of test and fitted result ( $\square$ : test result; red: $0.5 \mathrm{~Hz}$; black: $0.1 \mathrm{~Hz}$; blue: $0.03 \mathrm{~Hz}$; —: Model 1; - - -: Model 2; - . -: Model 3).

algorithm presented by Stron and Price is a relatively new population based stochastic optimization approach [24]. As a branch of evolution algorithm, DE has a similar theory to other evolution algorithms, including population initialization, mutation, crossover, and selection operation. The crucial difference is that DE uses the difference of randomly sampled pairs of vectors in the population for its mutation operator and other evolutionary algorithms use predetermined probability distribution functions. DE algorithm has been attracting increasing attention for it is simple, fast, and robust.

4.5. Comparison with Conventional Model. Compared to conventional creep-fatigue damage mechanical model, two modifications have been made in this paper. First, frequency modification was introduced into pure fatigue evolution equation. Second, nonlinear summation of damage increment was proposed. The comparison between the fitted results by modified model and conventional model was presented in Table 2 and Figure 8. Model 1 was the proposed model, Model 2 turned nonlinear damage increment summation back to linear, that is, $C \equiv 1$, and Model 3 cleared away frequency modification from fatigue evolution equation. As shown by the results, the fitted result of Model 1 agreed best with tested result; Model 3 agreed worst. The difference between Model 2 and Model 1 was quite small; it was because in all tests of this paper creep damage was quite small compared to fatigue damage, and the values of $C$ in Model 1 were close to 1 . The comparison also indicated that fatigue strength rate dependence contributed largely to fatigue life frequency sensitivity of Plexiglas Resist 45.

\section{Conclusion}

Tensile fatigue tests at three frequencies $(0.03,0.1$, and $0.5 \mathrm{~Hz})$ were performed in MTS809, and the results showed that fatigue life increases with frequency. Frequency had little 
TABLE 2: Comparison of equation and $R^{2}$ of three models.

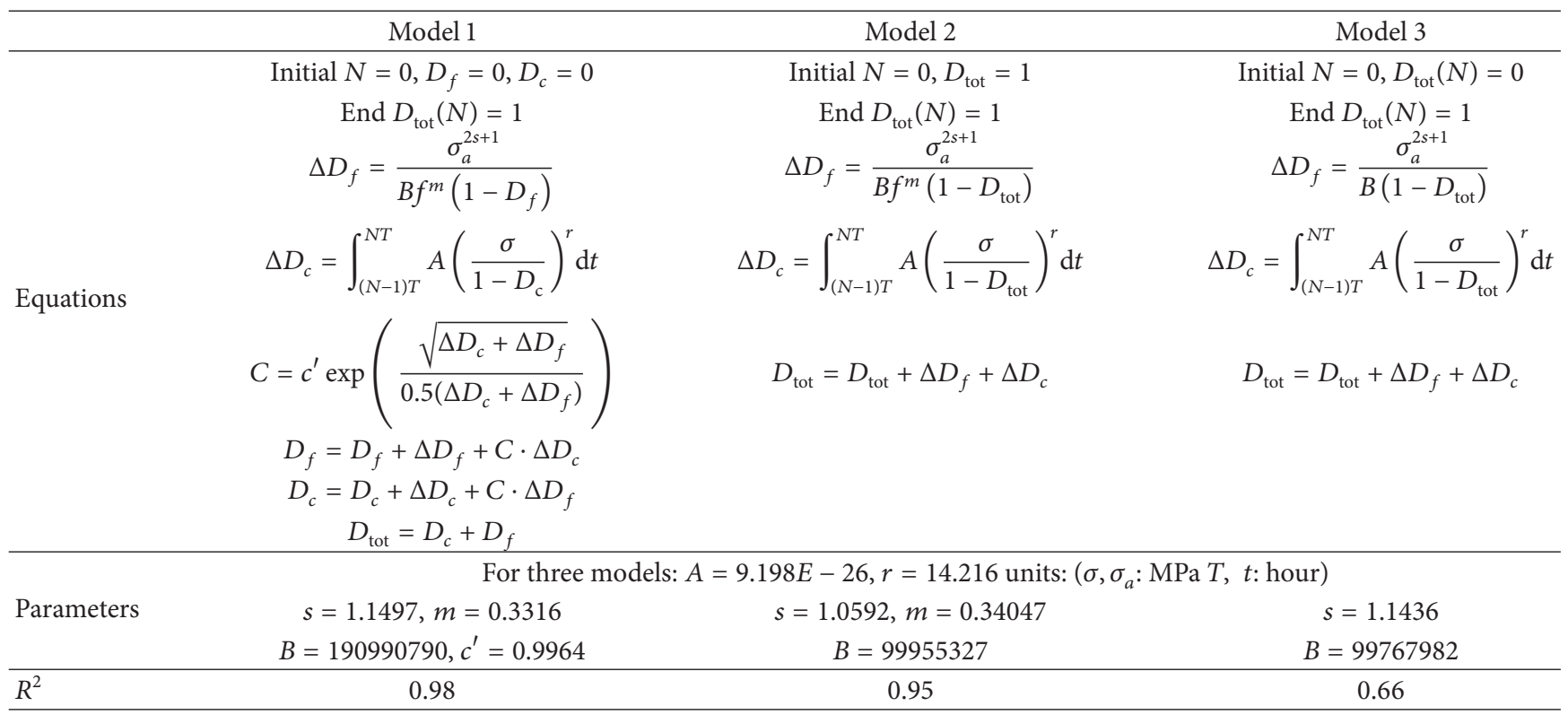

impact on cyclic creep rate and the area within hysteresis loop, but modulus increased with frequency.

Based on the analysis of test data and the viscoelastic mechanical properties of Plexiglas, creep damage along with creep-fatigue interaction, and fatigue strength rate-dependence, these two factors should be concerned with the life prediction. A creep-fatigue damage model including damage increment nonlinear summation and frequency modification was presented for life analysis. The comparison of test and calculation result showed the capability of the proposed model to describe the fatigue life of Plexiglas Resist 45 at different frequencies.

\section{Disclosure}

Aifeng Huang is the first author.

\section{Conflict of Interests}

The authors declare that there is no conflict of interests regarding the publication of this paper.

\section{Acknowledgment}

This work was supported by National Natural Science Foundation of China (no. 51275241).

\section{References}

[1] Editorial Committee of Fatigue and Fracture Atlas of PMMA, Fatigue and Fracture Atlas of PMMA, Science Press, Beijing, China, 1987.

[2] W. M. Cheng, G. A. Miller, J. A. Manson, R. W. Hertzberg, and L. H. Sperling, "Mechanical behaviour of poly (methyl methacrylate)-part 2: the temperature and frequency effects on the fatigue crack propagation behaviour," Journal of Materials Science, vol. 25, no. 4, pp. 1924-1930, 1990.

[3] W. Liu and X. J. Yang, "Damage evolution with growing cyclic creep and life prediction of MDYB-3 PMMA," Fatigue \& Fracture of Engineering Materials \& Structures, vol. 36, no. 6, pp. 483-491, 2012.

[4] J.-H. Jia, Y.-Z. Li, and J. Xiao, "Study on fatigue crack propagation behavior of YB-MD-3 PMMA plates," Journal of Aeronautical Materials, vol. 26, no. 5, pp. 109-112, 2006.

[5] L. Feng, Z. Yin, S. Gan et al., "Fatigue crack propagation of PMMA," Polymer Materials Science and Engineering, vol. 16, no. 6, pp. 121-123, 2000.

[6] C.-L. Luo, W.-X. Yao, and Z.-L. Zhang, "Separation model of fatigue-damage and creep-damage of polymethyl methacrylate for fatigue life prediction," Journal of Nanjing University of Aeronautics \& Astronautics, vol. 37, no. 6, pp. 741-744, 2005.

[7] H. Wang, Y. Wang, X. Yi, and J. Zhuang, "Frequency response regularity of cyclic toughness and S-N curve in polymethry methacrylate," Mechanical Science and Technology for Aerospace Engineering, vol. 30, no. 5, pp. 699-702, 2011.

[8] W. M. Cheng, G. A. Miller, J. A. Manson, R. W. Hertzberg, and L. H. Sperling, "Mechanical behaviour of poly(methyl methacrylate)-part 1: tensile strength and fracture toughness," Journal of Materials Science, vol. 25, no. 4, pp. 1917-1923, 1990.

[9] H. Wu, G. Ma, and Y. Xia, "Experimental study on mechanical properties of PMMA under unidirectional tensile at low and intermediate strain rates," Journal of Experimental Mechanics, vol. 20, no. 2, pp. 193-198, 2005.

[10] M. D. Skibo, R. W. Hertzberg, and J. A. Manson, “The effect of temperature on the frequency sensitivity of fatigue crack propagation in polymers," Fracture, vol. 3, pp. 1127-1133, 1977.

[11] A. Bernasconi and R. M. Kulin, "Effect of frequency upon fatigue strength of a short glass fiber reinforced polyamide 6: a superposition method based on cyclic creep parameters," Polymer Composites, vol. 30, no. 2, pp. 154-161, 2009. 
[12] Y. Hu, J. Summers, A. Hiltner, and E. Baer, "Correlation of fatigue and creep crack growth in poly(vinyl chloride)," Journal of Materials Science, vol. 38, no. 4, pp. 633-642, 2003.

[13] M. Parsons, E. V. Stepanov, A. Hiltner, and E. Baer, "Effect of strain rate on stepwise fatigue and creep slow crack growth in high density polyethylene," Journal of Materials Science, vol. 35, no. 8, pp. 1857-1866, 2000.

[14] F. Xu, Polymer Material Mechanics Test, Science Press, Beijing, China, 1988.

[15] A. M. Vinogradov and S. Schumacher, "Cyclic creep of polymers and polymer-matrix composites," Mechanics of Composite Materials, vol. 37, no. 1, pp. 29-34, 2001.

[16] H. Wu, Damage Mechanics, National Defence Industry Press, Beijing, China, 1990.

[17] J. Shi, "Measurement of elastic modulus of plexiglass," Journal of Suzhou College of Education, no. 3, pp. 83-84, 1996.

[18] J. Lemaitre and J. L. Chaboche, Solid Mechanics of Materials, translated by T. Yu, Y. Wu, National Defence Industry Press, Beijing, China, 1997.

[19] Z. Song, W. Huang, and M. Liu, "Study of the mutual damage of polystyrene both in fatigue and creep," Polymer Materials Science and Engineering, vol. 19, no. 1, pp. 188-190, 2003.

[20] X. J. Yang, C. L. Chow, and K. J. Lau, "A unified viscoplastic fatigue damage model for $63 \mathrm{Sn}-37 \mathrm{~Pb}$ solder alloy under cyclic stress control," International Journal of Damage Mechanics, vol. 12, no. 3, pp. 225-243, 2003.

[21] H. Xiao, X. Li, Y. Yan, N. Liu, and Y. Shi, "Damage behavior of $\mathrm{SnAgCu}$ solder under thermal cycling," Rare Metal Materials and Engineering, vol. 42, no. 2, pp. 221-226, 2013.

[22] L. M. Kachanov, Introduction to Continuum Damage Mechanics, Martinus Nijhoff Publishers, Dordrecht, The Netherlands, 1986.

[23] J.-L. Chaboche and F. Gallerneau, "An overview of the damage approach of durability modelling at elevated temperature," Fatigue and Fracture of Engineering Materials \& Structures, vol. 24, no. 6, pp. 405-418, 2001.

[24] R. Storn and K. Price, "Differential evolution-a simple and efficient Heuristic for global optimization over continuous spaces," Journal of Global Optimization, vol. 11, no. 4, pp. 341-359, 1997. 


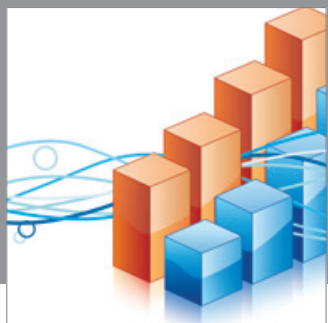

Advances in

Operations Research

mansans

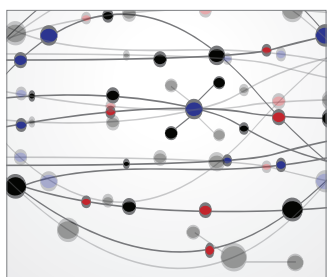

The Scientific World Journal
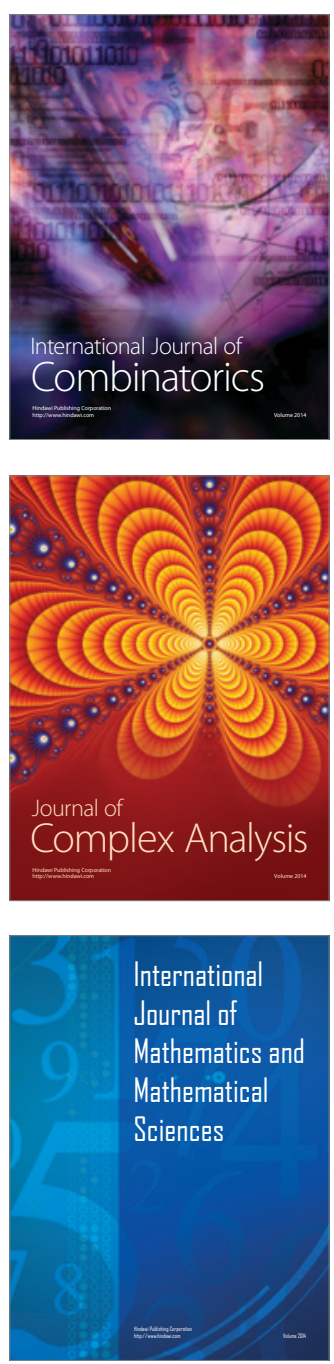
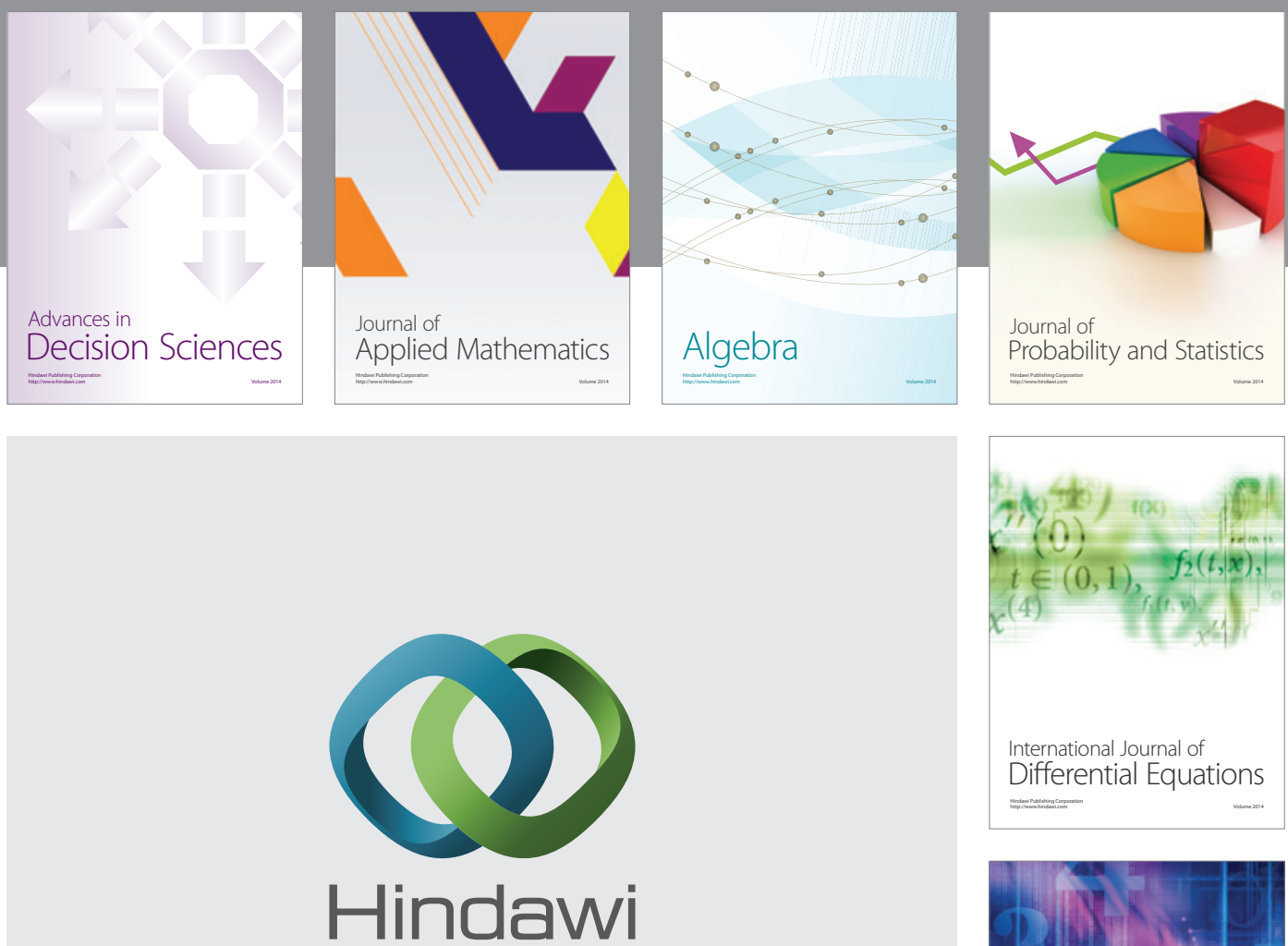

Submit your manuscripts at http://www.hindawi.com
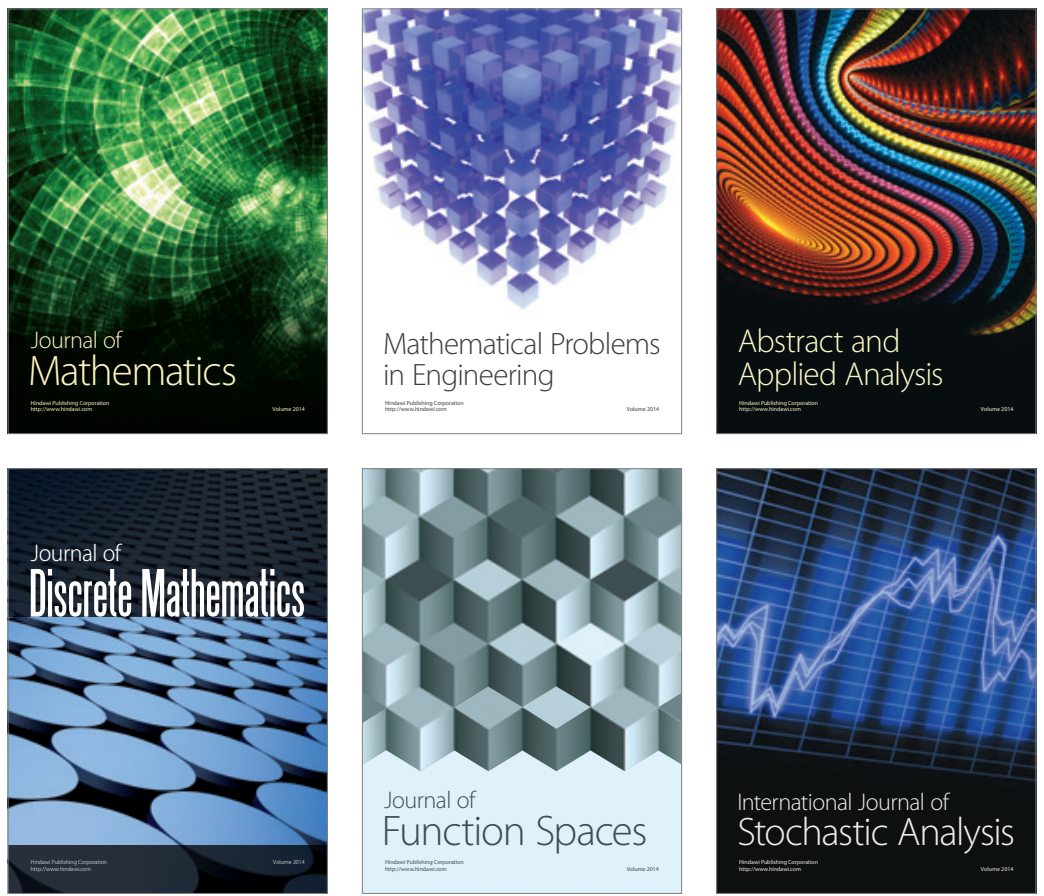

Journal of

Function Spaces

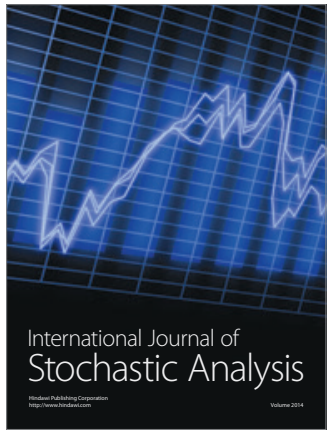

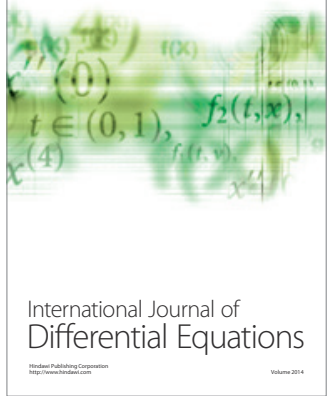
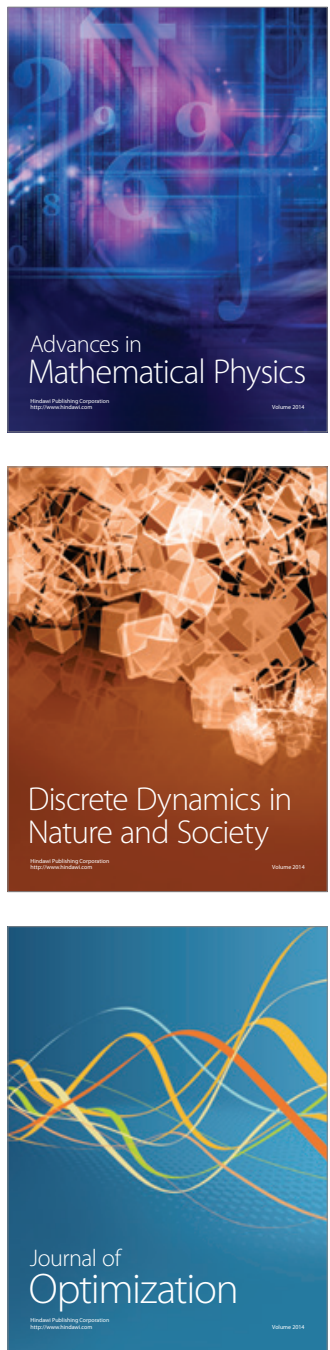